\title{
Emotional responses and coping strategies of medical students during the COVID-19 pandemic
}

Sayyada IfrahNaaz, Medicalstudent, RanaM. Hussein, Medicalstudent, Hiba B. Khan, Medicalstudent, MohamedM. Hussein, Medicalstudent, Shoukat A. Arain, MBBS, PhD.

\section{ABSTRACT}

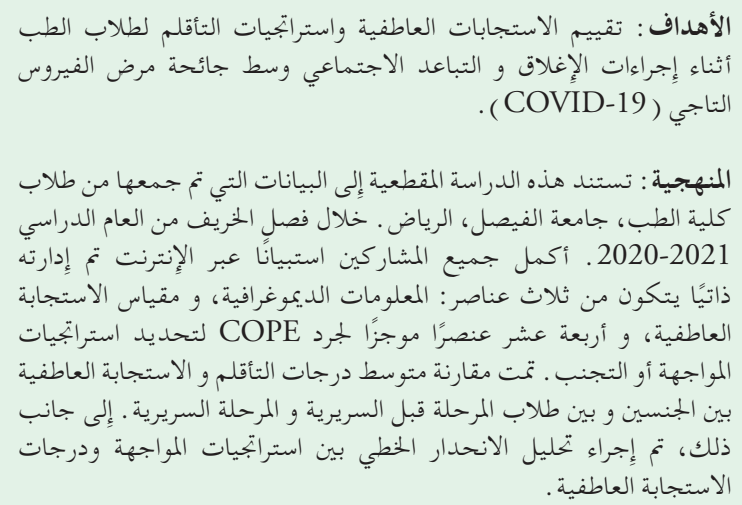

Objectives: To assess the emotional responses and coping strategies of medical students during the lockdown and social distancing measures implemented during the coronavirus disease -19 (COVID-19) pandemic.

Methods: This cross-sectional study is based on data collected from undergraduate medical students at the College of Medicine, Alfaisal University Riyadh, Saudi Arabia, during the fall semester of academic year 2020-2021. All the participants completed a self-administered online questionnaire consisting of 3 parts: demographic information, emotional response scale, and 14-item, adapted brief coping orientation to problems experienced inventory to determine the use of avoidant or approach coping strategies. Coping and emotional response scores were compared using t-test. Linear regression analysis was also performed.

Results: A total of 261 students from all years were included. Overall scores were higher for avoidant coping strategies. The use of avoidant coping strategies was significantly higher in females $(p=0.03)$ and in preclinical students $(p<0.001)$. Preclinical students had a higher mean score for anger $(p=0.002)$. Conversely, students in the clinical phase had higher scores for anxiety $(p=0.005)$ and sadness $(p=0.027)$. The regression analysis of emotional responses and coping strategies suggests that avoidant coping is a predictor of anger $(p=0.003)$ and sadness $(p=0.005)$.

Conclusion: Interventions to train medical students in the use of more productive and effective coping strategies may reduce negative emotional responses linked to the present COVID-19 pandemic and in the future.

Keywords: coping strategies, COVID-19, emotional responses, medical students, pandemic

Saudi Med J 2022; Vol. 43 (1): 61-66 doi: 10.15537/smj.2022.43.1.20210698

From the College of Medicine (Naaz, R. Hussein, Khan, M. Hussein); and from the Department of Pathology (Arain), College of Medicine, Alfaisal University, Riyadh, Kingdom of Saudi Arabia.

Received 31st August 2021. Accepted 3rd November 2021.

Address correspondence and reprint request to: Dr. Shoukat A. Arain, Department of Pathology, College of Medicine, Alfaisal University, Riyadh, Kingdom of Saudi Arabia. E-mail:sa_arain@hotmail.com ORCID ID: https://orcid.org/0000-0003-3347-6796 
$\mathrm{N}$ ovel coronavirus disease -19 (COVID-19) initially appeared in December 2019 in the city of Wuhan, China. Since then, it has rapidly spread all around the world, prompting the World Health Organization (WHO) to declare it a pandemic on March 11, 2020. In Saudi Arabia, the first case of COVID-19 was recorded on March 2, 2020, and subsequently, to limit the spread of the virus, sudden lockdown protocols, such as closing of educational institutions, shopping malls, and mosques were implemented in the country on March 9, 2020. ${ }^{1}$

Social distancing and lockdown have a negative effect on mental health and increase emotional responses of fear, stress, depression, and anxiety. Infectious disease outbreaks have psychological implications not only for people who have been infected but also for caregivers and healthcare workers. During previous infectious viral outbreaks, such as severe acute respiratory syndrome (SARS), people experienced a variety of emotional responses, including stress disorders, anxiety, fear, and anger. $^{2}$

Some groups are at greater risk of developing emotional symptoms. Numerous studies, from those dedicated to screening for psychopathological symptoms to those focused on university adaptation, have investigated the mental health of the university population. College students are known to suffer from depression, anxiety, and stress at a higher rate than other populations in normal times., ${ }^{3,4}$ Furthermore, the lockdown led to schools and universities adopting virtual learning as a means of instruction, which might have substantial repercussions for students' social and mental well-being.

When faced with adversities such as infectious viral outbreaks, people typically adopt coping methods, or ways to respond to the problem. Coping is a collection of psychological responses with the goal of preventing or reducing threat, harm, and loss as well as related discomfort. Individuals use a variety of coping mechanisms to minimize psychological suffering. Coping strategies generally are of 2 types: approach coping and avoidant coping. Approach coping aims to fix the problem or take steps to improve the status quo, whereas avoidant coping aims to mitigate the discomfort associated with traumatic circumstances. Using a set of "functional" methods in approach coping is linked to improved physical and mental health in the face of a variety of stressors. ${ }^{5}$ During the 2003 SARS pandemic,

Disclosure. Authors have no conflict of interests, and the work was not supported or funded by any drug company. research on university students revealed that coping was an essential mechanism to mitigate the negative effect of stressors on perceived mental and physical health. ${ }^{6}$ Furthermore, it was found that adults adopted fewer active techniques (focused on solving problems) and more avoidant coping techniques (focused on emotions) in response to SARS-related stressors. ${ }^{7}$

To effectively manage the present pandemic and to establish mental health response plans for the future, a better understanding of emotional responses and coping skills across the community are essential. From this perspective, there is a need to assess the mental health of medical students. The purpose of this study was to identify the emotional responses and coping strategies used by medical students and to determine the correlation between the used coping strategies and emotional responses.

Methods. In this cross-sectional study, data was collected, using convenience sampling, from undergraduate medical students at the College of Medicine, Alfaisal University Riyadh, Saudi Arabia, during the fall semester of academic year 2020-2021. Invitations to take part in the study were shared through personal and professional electronic contacts. All the participants who completed the online questionnaire and gave informed consent were included in the study. Participants who submitted an incomplete questionnaire were excluded. The study was conducted according to the guidelines of the Declaration of Helsinki and was approved by the Institutional Review Board (Ref No. IRB-20064, dated 11/10/2020).

The partially validated questionnaire consisted of 3 parts. In the first part, respondents answered demographic questions. In the second part, respondents rated their emotional responses (anxiety, fear, anger, and sadness) during the lockdown on a 5-point scale where $1=$ no emotion, and 5=strong emotion. In the third part of the questionnaire, the brief Coping Orientation to Problems Experienced (COPE) inventory was used to assess coping strategies. ${ }^{8}$ The brief-COPE inventory is a condensed version of the 60 -item COPE questionnaire. The brief-COPE is a 28-item, 4-point Likert scale that has been validated to assess the effectiveness of specific interventions in various contexts. ${ }^{10}$ The original inventory consists of 14 subscales, with 2 questions per subscale. However, since not all the questions were relevant to our study, we have selected one question per subscale that was most relevant to the COVID-19 context to include in our questionnaire. Thus, our adapted brief-COPE comprised 14 questions. Participants were asked to report on how frequently they used the strategy described in each question, where $1=$ never, and $4=$ always. The total score was calculated 
by adding the individual scores for each question falling under either an approach or avoidant coping strategy.

Statistical analysis. Data was analyzed using Statistical Package for the Social Sciences version 27 (IBM Corp., Armonk, NY, USA). Frequencies and percentages were calculated for all nominal variables. Mean coping and emotional response scores were compared for gender and phase of study (clinical or preclinical) using the independent samples t-test. Linear regression was used to analyze the correlation between coping strategies and emotional response scores. In all analyses, a $p$-value $<0.05$ was considered significant.

Results. The 261 study participants were undergraduate medical students. The mean age of the participants was 19.63 years (range: 17-24). Demographic data (Table 1) showed that a majority

Table 1 - Baseline characteristics of the study sample ( $\mathrm{N}=261)$.

\begin{tabular}{lc}
\hline Variables & $\mathbf{n}(\%)$ \\
\hline Gender & \\
Female & $141(54.0)$ \\
Male & $120(46.0)$ \\
Year of study & \\
First year & $60(23.0)$ \\
Second year & $39(14.9)$ \\
Third year & $41(15.7)$ \\
Fourth year & $65(24.9)$ \\
Fifth year & $56(21.5)$ \\
Overall scores, mean $\pm S D$ & \\
Avoidant coping & $20.65 \pm 1.80$ \\
Approach coping & $19.62 \pm 1.58$ \\
Anxiety & $3.45 \pm 1.00$ \\
Fear & $2.46 \pm 1.07$ \\
Anger & $3.14 \pm 1.37$ \\
Sadness & $2.15 \pm 1.11$ \\
\hline \multicolumn{2}{l}{} \\
\hline
\end{tabular}

of the participants were female (54\%). A fair representation from each academic year is evident. The mean score for avoidant coping strategies was higher than that for approach coping strategies. The mean emotional response scores, in ascending order, were: 2.15 for sadness, 2.46 for fear, 3.14 for anger, and 3.45 for anxiety.

Respondents' mean scores for coping strategies for each year are presented in Figure 1. Generally, the mean scores for avoidant coping across all years of study were higher than the means scores for approach coping. The mean scores for avoidant coping for students in first year and second year were higher the mean scores for avoidant coping for students in third, fourth, and fifth year.

The mean scores of anxiety, fear, anger, and sadness for each year of study are represented in a bar graph in Figure 2. Overall, the mean scores for anxiety and sadness were higher in fifth-year students, while the mean scores for fear were higher in first- and fourth-year students, and the mean scores for anger were higher in first year students.

Comparison of coping strategies and emotional responses between gender, and preclinical and clinical years of study is shown in Table 2. The mean scores of females were higher than those of males for avoidant coping $(p=0.03)$ and were significantly lower for approach coping $(p=0.035)$. However, no statistically significant difference was found in the scores for emotional responses between males and females. The avoidant coping scores of the preclinical students were significantly higher than those of the clinical students $(p<0.001)$. The scores for anger $(p=0.002)$ were significantly higher for students in the preclinical phase of study. However, the students in the clinical phase

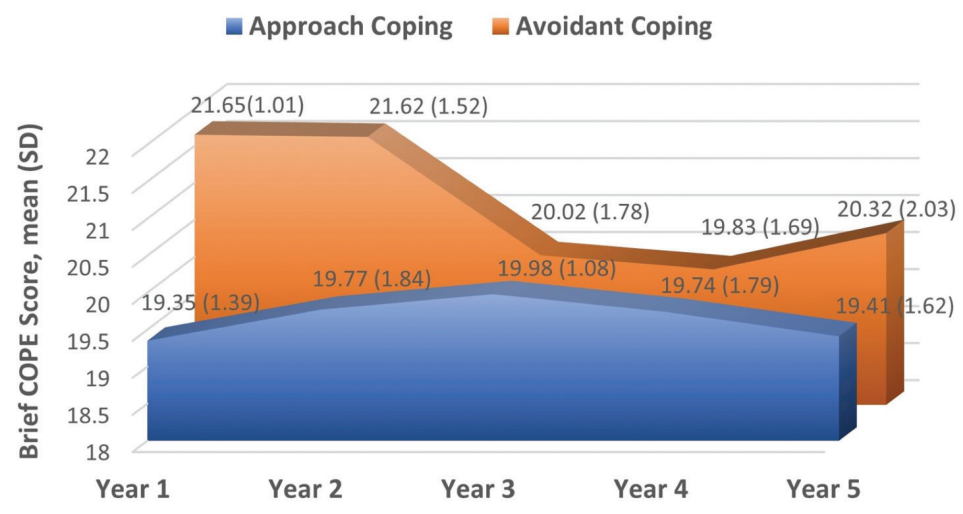

Figure 1 - Year-wise mean avoidant and approach coping scores. COPE: coping orientation to problems experienced, SD: standard deviation 


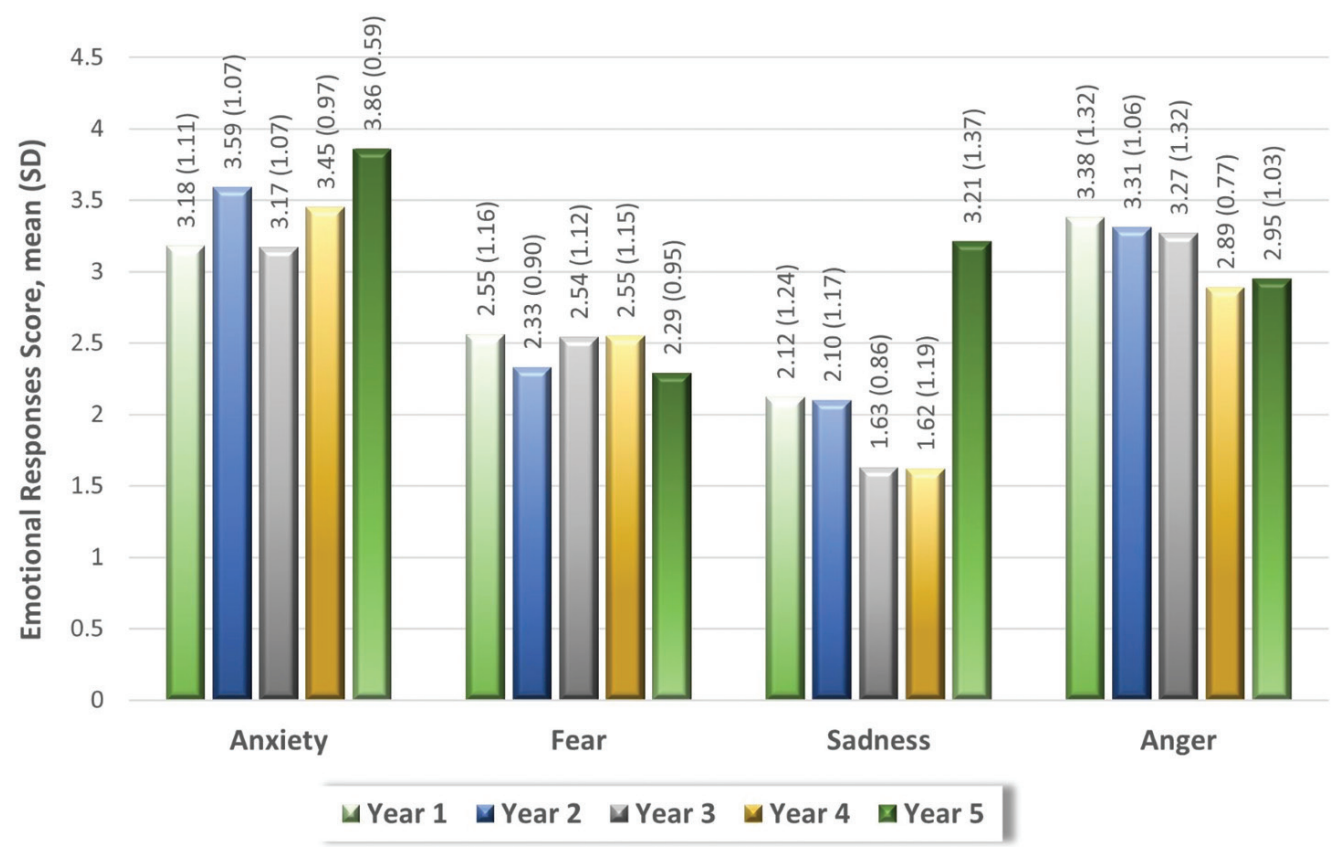

Figure 2 - Year-wise mean emotional response scores. SD: standard deviation

Table 2 - Comparison of coping strategies and emotional response scores for gender and phase of study (N=261).

\begin{tabular}{|c|c|c|c|c|c|c|}
\hline \multirow[t]{3}{*}{ Response } & \multicolumn{3}{|c|}{ Gender } & \multicolumn{3}{|c|}{ Phase of study } \\
\hline & Male & Female & $P$-value & Preclinical $^{\dagger}$ & Clinical $^{\ddagger}$ & $P$-value \\
\hline & \multicolumn{2}{|c|}{ mean $\pm S D$} & \multicolumn{4}{|c|}{ mean $\pm S D$} \\
\hline Avoidant coping & $20.38 \pm 2.03$ & $20.88 \pm 1.56$ & $0.030^{*}$ & $21.16 \pm 1.59$ & $20.06 \pm 1.86$ & $<0.001^{*}$ \\
\hline Approach coping & $19.84 \pm 1.44$ & $19.43 \pm 1.67$ & $0.035^{*}$ & $19.65 \pm 1.47$ & $19.59 \pm 1.71$ & 0.748 \\
\hline Anxiety & $3.44 \pm 1.04$ & $3.46 \pm 0.97$ & 0.876 & $3.29 \pm 1.10$ & $3.64 \pm 0.84$ & $0.005^{*}$ \\
\hline Fear & $2.34 \pm 0.99$ & $2.56 \pm 1.12$ & 0.096 & $2.49 \pm 1.08$ & $2.43 \pm 1.06$ & 0.674 \\
\hline Anger & $3.16 \pm 1.10$ & $3.12 \pm 1.13$ & 0.786 & $3.33 \pm 1.24$ & $2.92 \pm 0.90$ & $0.002^{*}$ \\
\hline Sadness & $2.07 \pm 1.36$ & $2.22 \pm 1.38$ & 0.37 & $1.97 \pm 1.13$ & $2.36 \pm 1.59$ & $0.027^{*}$ \\
\hline
\end{tabular}

SD: standard deviation, ${ }^{\dagger}$ year $1-3,{ }^{\ddagger}$ year 4 and $5, " p$-value indicates that there is a significant difference

of study had significantly higher scores for anxiety $(p=0.005)$ and sadness $(p=0.027)$ than the preclinical students.

To learn about the relationship between coping strategies and emotion scores, a linear regression analysis was performed. The dependent variable in the regression analysis was emotion scores, while the independent variables were the 2 coping methods. Only avoidant coping was included in the regression equation of anger and sadness. A significant regression equation was found $(F[1,259]=9.16 ; p=0.003)$ with an $\mathrm{R}^{2}$ of 0.03 (Table 3 ). The $\mathrm{R}$-squared value indicates that avoidant coping account for $3 \%$ variance of anger. Avoidant coping was entered in the regression equation of sadness, and an $\mathrm{R}^{2}$ of 0.03 (F $\left.[1,259]=8.19 ; p=0.005\right)$ indicated that the regression equation was significant, and avoidant coping explains $3 \%$ of the variation in sadness.

Discussion. It is generally recognized that infectious disease outbreaks cause uncertainty and insecurity as well as poor rational thinking, resulting in psychological distress with symptoms of mental ill health. ${ }^{11}$ This study revealed that, during the pandemic, medical students were more likely to adopt avoidant coping strategies and experienced, in rising order of intensity, sadness, fear, anger, and anxiety. Mean avoidant coping scores were significantly higher in females and in the preclinical phase of study. Moreover, students with higher avoidant coping scores also had higher anger 
Table 3 - Regression analysis of emotional responses during COVID-19 as a function of coping strategies.

\begin{tabular}{|c|c|c|c|c|c|c|c|c|c|c|c|c|}
\hline \multirow[t]{3}{*}{ Independent variable } & \multicolumn{12}{|c|}{ Dependent variables } \\
\hline & \multicolumn{3}{|c|}{ Anxiety } & \multicolumn{3}{|c|}{ Fear } & \multicolumn{3}{|c|}{ Anger } & \multicolumn{3}{|c|}{ Sadness } \\
\hline & Beta & $\mathrm{T}$ & $P$-value & Beta & $\mathrm{T}$ & $P$-value & Beta & $\mathrm{T}$ & $P$-value & Beta & $\mathrm{T}$ & $P$-value \\
\hline Avoidant coping & 0.1 & 1.1 & 0.3 & 0.1 & 1.5 & 0.2 & 0.2 & 3 & $0.003^{*}$ & 0.2 & 2.9 & $0.005^{*}$ \\
\hline Approach coping & -0.1 & -1.1 & 0.3 & -0.1 & -1.6 & 0.1 & 0 & -0.4 & 0.717 & -0.1 & -1.4 & 0.178 \\
\hline
\end{tabular}

and sadness scores. These results are consistent with existing studies. Studies conducted in the early stages of the COVID-19 pandemic indicated that younger adults and females showed more depression and anxiety symptoms than older age groups. ${ }^{12,13}$ Similarly, a major nationwide study in the United Kingdom reported that mental health decreased dramatically after one month into the lockdown, particularly among undergraduate students and women. ${ }^{14}$

In the past year, the learning structure of preclinical years changed dramatically at our university as digital platforms replaced in-person learning. The use of novel online teaching methods as an urgent response to the pandemic, representing a transition to the "new normal" of delivering preclinical medical education, comes with both concerns and opportunities. Our survey revealed that, compared to the clinical phase, preclinical students adopted negative coping strategies more. The results also indicated that the preclinical medical students experienced significantly higher levels of anger. The switch from on-campus learning to remote learning resulted in isolation, stress, loss of focus, less engagement with classmates, and distraction due to family matters. ${ }^{15}$ Also, access to practical labs as well as models, skeletons, and histology labs was restricted due to the sudden lockdown. ${ }^{16-18}$ Concerns have also been expressed over prolonged screen time, the lack of social engagement, and the lack of face-to-face verbal and nonverbal communication. ${ }^{15,19}$

The results also indicate that students in the clinical phase of medical education had higher scores for anxiety and sadness than those in the preclinical phase. During the final 2 years of medical school, students rotate through core clerkships, and they receive intense clinical training. In response to the COVID-19 outbreak, clinical placements in hospitals, general practices, and community settings were limited. Consequently, students' development of clinical skills may have been affected. Moreover, many clinical students were unable to participate in co-curricular activities such as research, which is important in distinguishing candidates who will go on to apply to specialty training programs in the future. These restricted opportunities may induce anxiety and thus, sadness in students about their career progression. ${ }^{20}$

In general, women are known to report more depression, anxiety, and isolation than men. During the SARS outbreak, women sought psychological treatment more often than men, with the majority of consultations focusing on mental distress. ${ }^{21}$ Our study supports these findings by revealing that female students adopted more negative coping strategies linked with poorer psychological health outcomes than male students. These findings not only are consistent with pre-pandemic statistics indicating more mental health problems among women but also reflect the greater impact of the current pandemic on the mental health of female students. ${ }^{21,22}$

We conducted a linear regression analysis to investigate the effect of coping strategies on emotions and found that adopting avoidant coping is associated with greater sadness and anger. However, avoidant coping strategies appear to be predictive of these emotional responses only to a certain extent, with a possibility of other variables, not explored in this study. Our findings are consistent with earlier studies showing that coping skills training reduced sadness, anxiety, and stress in adolescents with type 1 diabetes and in drug addicts. ${ }^{23,24}$

Even if the number of COVID-19 infections decreases and restrictions are relaxed, the pandemic's negative impact on people's mental health may persist. Following SARS, several negative mental health symptoms, including stress, sadness, anxiety, and despair, persisted long after the restrictions were lifted. ${ }^{2}$ Therefore, we recommend that medical students be trained in effective coping techniques through emotional management methods.

Study limitations. The data was collected using an online questionnaire with a possibility of selection bias as participants self-selected themselves. An important limitation is the use of a simplified self-perceived emotional response scale instead of a pre-validated standard tool to measure respondents' emotional 
responses. However, we were able to demonstrate a correlation between self-reported emotional responses and coping strategies. Another limitation is that the respondents were from a single university. More research is needed to evaluate whether the current findings are broadly generalizable to other students and young adults in general as people's experiences outside of the educational system may differ significantly.

In conclusion, medical students generally used more avoidant coping strategies as compared to approach coping strategies. It was further revealed that females and preclinical students adopted more avoidant coping strategies, and avoidant coping was a predictor of anger and sadness. Students in the clinical phase of study had higher levels of anxiety and sadness, whereas students in the preclinical phase experienced more anger.

Training to increase the use of more productive and effective coping strategies may reduce negative emotional responses linked to the present COVID-19 pandemic. Furthermore, additional research is required identify the variables of mental distress and to provide a more comprehensive picture of the situation.

Acknowledgment. The authors gratefully acknowledge SCRIBENDI (www.scribendi.com) for English language editing.

\section{References}

1. Bedford J, Enria D, Giesecke J, Heymann DL, Ihekweazu C, Kobinger G, et al. COVID-19: towards controlling of a pandemic. Lancet 2020; 395: 1015-1018.

2. Reynolds DL, Garay JR, Deamond SL, Moran MK, Gold W, Styra R. Understanding, compliance and psychological impact of the SARS quarantine experience. Epidemiol Infect 2008; 136: 997-1007.

3. Brenneisen Mayer F, Souza Santos I, Silveira PS, Itaqui Lopes $\mathrm{MH}$, de Souza AR, Campos EP, et al. Factors associated to depression and anxiety in medical students: a multicenter study. BMC Med Educ 2016; 16: 282.

4. Saraswathi I, Saikarthik J, Senthil Kumar K, Madhan Srinivasan K, Ardhanaari M, Gunapriya R. Impact of COVID-19 outbreak on the mental health status of undergraduate medical students in a COVID-19 treating medical college: a prospective longitudinal study. PeerJ 2020; 8: e10164.

5. Duangdao KM, Roesch SC. Coping with diabetes in adulthood: a meta-analysis. J Behav Med 2008; 31: 291-300.

6. Main A, Zhou Q, Ma Y, Luecken LJ, Liu X. Relations of SARS-related stressors and coping to Chinese college students' psychological adjustment during the 2003 Beijing SARS epidemic. J Couns Psychol 2011; 58: 410-423.

7. Gan Y, Liu Y, Zhang Y. Flexible coping responses to severe acute respiratory syndrome-related and daily life stressful events. Asian J Soc Psychol 2004; 7: 55-66.

8. Carver CS. You want to measure coping but your protocol's too long: consider the brief COPE. Int J Behav Med 1997; 4: 92-100.
9. Carver CS, Scheier MF, Weintraub JK. Assessing coping strategies: a theoretically based approach. J Pers Soc Psychol 1989; 56: 267-283.

10. Meyer B, Johnson SL, Winters R. Responsiveness to threat and incentive in bipolar disorder: relations of the BIS/BAS scales with symptoms. J Psychopathol Behav Assess 2001; 23: 133-143.

11. Mukhtar S. Psychological health during the coronavirus disease 2019 pandemic outbreak. Int J Soc Psychiatry 2020; 66: 512-516.

12. Ozamiz-Etxebarria N, Dosil-Santamaria M, PicazaGorrochategui M, Idoiaga-Mondragon N. Stress, anxiety, and depression levels in the initial stage of the COVID-19 outbreak in a population sample in the northern Spain. Cad Saude Publica 2020; 36: e00054020.

13. Gao W, Ping S, Liu X. Gender differences in depression, anxiety, and stress among college students: a longitudinal study from China. J Affect Disord 2020; 263: 292-300.

14. Pierce M, Hope H, Ford T, Hatch S, Hotopf M, John A, et al. Mental health before and during the COVID-19 pandemic: a longitudinal probability sample survey of the UK population. Lancet Psychiatry 2020; 7: 883-892.

15. Liang ZC, Ooi SBS, Wang W. Pandemics and their impact on medical training: lessons from Singapore. Acad Med 2020; 95: 1359-1361.

16. Gaur U, Majumder MAA, Sa B, Sarkar S, Williams A, Singh K. Challenges and opportunities of preclinical medical education: COVID-19 crisis and beyond. SN Compr Clin Med 2020: 1-6.

17. Longhurst GJ, Stone DM, Dulohery K, Scully D, Campbell T, Smith CF. Strength, weakness, opportunity, threat (SWOT) analysis of the adaptations to anatomical education in the United Kingdom and Republic of Ireland in response to the covid-19 pandemic. Anat Sci Educ 2020; 13: 301-311.

18. Pather N, Blyth P, Chapman JA, Dayal MR, Flack NAMS, Fogg QA, et al. Forced disruption of anatomy education in Australia and New Zealand: an acute response to the covid-19 pandemic. Anat Sci Educ 2020; 13: 284-300.

19. Gallagher TH, Schleyer AM. "We signed up for this!" - student and trainee responses to the covid-19 pandemic. $N$ Engl J Med 2020; 382: e96.

20. Rose S. Medical student education in the time of COVID-19. JAMA 2020; 323: 2131-2132.

21. Altemus M, Sarvaiya N, Neill Epperson C. Sex differences in anxiety and depression clinical perspectives. Front Neuroendocrinol 2014; 35: 320-330.

22. Liu CH, Stevens C, Conrad RC, Hahm HC. Evidence for elevated psychiatric distress, poor sleep, and quality of life concerns during the COVID-19 pandemic among U.S. young adults with suspected and reported psychiatric diagnoses. Psychiatry Res 2020; 292: 113345.

23. Habibi Z, Tourani S, Sadeghi H, Abolghasemi A. Effectiveness of stress management skill training on the depression, anxiety and stress levels in drug addicts after drug withdrawal. Int $J$ High Risk Behav Addict 2013; 2: 82-86.

24. Edraki M, Rambod M, Molazem Z. The effect of coping skills training on depression, anxiety, stress, and self-efficacy in adolescents with diabetes: a randomized controlled trial. Int J Community Based Nurs Midwifery 2018; 6: 324-333. 